\title{
The Immune-Modulatory Properties of iPSC-Derived Antigen-Presenting Cells
}

\author{
Mania Ackermann ${ }^{\text {a Anna Christina Dragon }}{ }^{\mathrm{b}}$ Nico Lachmann ${ }^{\mathrm{a}}$ \\ a Institute of Experimental Hematology, RG Translational Hematology of Congenital Diseases, REBIRTH - Research

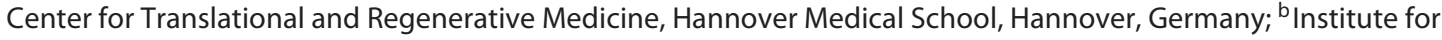 \\ Transfusion Medicine and Transplant Engineering, Hannover Medical School, Hannover, Germany
}

\section{Keywords}

Antigen presentation - Macrophage - Dendritic cells . Induced pluripotent stem cells · Cell therapy

\begin{abstract}
Antigen-presenting cells (APCs), such as dendritic cells (DCs) and macrophages, are important regulators of the immune system, as they connect the innate and adaptive immunity by critically regulating T-cell responses. Thus, APCs are involved in both tissue homeostasis and tolerance, but also coordinate immune responses in case of infection and inflammation. Primary APCs are commonly generated from peripheral blood-derived monocytes and have been used as cell therapeutics in several (pre-)clinical settings, e.g., immune oncology, however, with varying efficiency. One promising alternative to study antigen presentation in vitro and to develop novel cell-based therapies are induced pluripotent stem cells (iPSCs). IPSCs can nowadays be generated from a variety of different cell types using several refined reprogramming techniques. Given their unlimited proliferation and differentiation potential, they hold great promise for regenerative medicine, and recently, first iPSC derivatives have found their way into first clinical studies for cell-based therapies. In this review article, we will give a brief overview of current methods for the generation and applications of primary APCs, but also specifically focus on different strategies for the generation of defined subsets of DCs and macrophages from human PSCs. Moreover, we will highlight the potential but also hurdles for the clinical translation of iPSCderived APCs.

(c) 2020 S. Karger AG, Basel
\end{abstract} poietic cell types, which orchestrate not only host defense but also fulfill various other functions, such as maintaining tissue homeostasis or mediating cellular tolerance. Disturbances in this finely balanced network of cell-cell interaction or interference with the reactivity or production of cell-extrinsic modulators may lead to unwanted side effects and can cause a variety of diseases with varying clinical outcomes. Given the interplay of hematopoietic cells of the innate and adaptive immunity, both macrophages and dendritic cells (DCs) have been described as two critical cellular players in mediating and regulating $\mathrm{T}$-cell responses. Thus, a variety of strategies are underway to directly target these professional antigen-presenting cells (APCs) in order to develop new therapeutic strategies to counteract different disease entities, such as allergies, autoimmune disorders, graft-versus-host disease, cancers, and many others. While the investigation or validation of new immunomodulatory drugs by sophisticated in vitro (co-)culture systems is only one approach, also the emerging field of cellular immunotherapies and the development of innovative adoptive cell transfer regimens opens new therapeutic options. To pave the way for such seminal (pre-)clinical approaches, both macrophages and DCs are typically generated from peripheral blood-derived $\mathrm{CD}_{1} 4^{+}$monocytes. Recently, however, the technology of induced pluripotent stem cells (iPSCs) has introduced a promising alternative strategy for the generation of APCs. In fact, iPSCs can be generated from various cell types and donors and their de- 
rivatives can be used either in an autologous or allogeneic context. Moreover, iPSC can be extensively genetically manipulated in vitro, further highlighting their attractiveness for regenerative medicine.

While previous studies could already demonstrate the generation of specific subsets of macrophages and DCs from human iPSCs, this review will provide a brief overview of different differentiation strategies and recent insights into the immunomodulatory properties of iPSCderived APCs. In the first part, we will give a short background about primary APCs and introduce different subtypes as well as ex vivo differentiation strategies. In the second part, we will focus on different methods to generate DCs and macrophages from human PSCs. Given the embryonic developmental background of iPSC-derived macrophages and DCs, also, their functional and transcriptomic fingerprint compared to their in vivo counterparts will be described. Finally, we will highlight seminal adoptive transfer approaches of primary as well as iPSCderived APCs to enhance drug development and to facilitate the field of transfusion medicine.

\section{The Status Quo of Studying Antigen Presentation in vitro and in vivo}

Myeloid, professional APCs that can prime naïve T cells are DCs and macrophages [1]. Although APCs are critical modulators for the adaptive immune system, the distinct definition and function of specific APC subsets and how those cells precisely control primary T-cell fate and activation remains largely unexplored, emphasizing the need for suitable models to study basic APC biology.

\section{Dendritic Cells}

DCs can be classified into conventional DCs 1 and 2 ( $\mathrm{cDC} 1$ and $\mathrm{cDC} 2$ ), plasmacytoid DCs (pDCs), monocytederived DCs (moDCs), and Langerhans cells (LCs). cDCs represent a classical morphology and capture a large variety of antigens in the periphery and subsequently present those to $\mathrm{T}$ cells in lymphoid organs $[2,3]$. Thereby, cDC1 are highly capable of cross-presentation without further stimulation enabling activation of CD8-positive T cells, whereas $\mathrm{CDC} 2$ show a wide capability to prime CD4-positive T cells [4]. cDC1 as well as pDCs, a more rare subset with a plasma cell morphology, are specialized to release high amounts of interferons following sensing of viral nucleic acids. LCs, known also as a subset within the family of tissue resident macrophages (TRM), are located in the basal epidermis and other epithelia, maintain tolerance under steady-state and respond towards intracellular pathogens and viruses under inflammatory conditions. However, the main population of DCs appearing in inflammation are moDCs, which have high expansion properties and are involved in many inflammatory diseases.

To study properties and function of DCs, primary human DCs are predominantly generated from human peripheral blood-derived monocytes. Other DC subsets represent such a rare population in the peripheral blood that they cannot be harvested in larger quantities. Moreover, these cells are postmitotic cells and cannot efficiently be expanded in culture. Thus, DCs for (pre-)clinical evaluation are commonly generated from peripheral blood-derived $\mathrm{CD}_{14}{ }^{+}$monocytes by the addition of granulocyte-macrophage colony-stimulating factor (GM-CSF) and interleukin (IL)-4 for 5-7 days yielding high numbers of moDCs. Advancing the functionality of in vitro-generated DCs, the cells can be genetically modified at different differentiation stages using, for example, lentiviral overexpression of specific cytokines or also antigens which are subsequently presented to tumor and virus-infected cells for use as vaccines [5]. As DCs develop from hematopoietic stem and progenitor cells in vivo, alternatively, also proliferative $\mathrm{CD} 34^{+}$cells derived from cord blood or mobilized peripheral blood samples can be used to produce DCs by addition of cytokines, such as FLT3 ligand (Flt3-L), stem cell factor (SCF), GM-CSF, IL4 , and tumor necrosis factor- $\alpha[6,7]$. Of note, the generated DCs can be further matured and, with varying efficiency, also differentiated in distinct DC subsets including cDCs, pDCs, and LCs by addition of specific cytokine cocktails allowing to choose the appropriate subset for the intended use [6]. Similarly, micro-RNAs have been applied to further direct the fate of DCs into specific subsets [8].

For preclinical evaluation of APCs, several mouse models have been applied, including genetically modified strains that allow for the ablation of specific DC subsets (reviewed in [9]). To reveal the functional interplay with other immune cells following presentation of distinct antigens in vivo, also adoptive transfer of antigen-loaded DCs or targeted delivery of specific antigens to DCs mediated by DC-specific antibodies have been utilized. Limitations of these resource-intensive, low-throughput, and costly murine in vivo models are quickly reached when the research focus shifts from basic biology to (pre-)clinical evaluation of different drugs or large compound screens. Moreover, these murine models do not necessarily reflect all facets of human DC and macrophage physiology. For specific aspects of human DC or macrophage biology, myeloid leukemia-derived cell lines or primary leukemia-derived cells $\left(\mathrm{DC}_{\text {leu }}\right)$ can be differentiated towards the desired phenotype providing cells in large numbers and extending tools for genetic manipulation [10-12]. However, none of these cell lines fully recapitulates all features of primary human APCs. 


\section{Macrophages}

Like DCs, human macrophages can easily be generated from human peripheral blood $\mathrm{CD} 14^{+}$monocytes or $\mathrm{CD} 34^{+}$hematopoietic stem and progenitor cells by the addition of lineage instructive cytokines, such as M-CSF, GM-CSF, and IL-3 [13, 14]. Although macrophages are mainly associated with their antimicrobial activity exerted by sensing, phagocytosing, and killing of bacteria as well as secretion of pro- and anti-inflammatory cytokines and chemokines to modulate the immune system, they also represent an important member of the class of APCs. Indeed, an early study suggests that macrophages can present antigens to naïve $\mathrm{CD} 8^{+} \mathrm{T}$ cells in similar efficiency to moDCs [15]. Moreover, specific macrophage subsets (e.g., CD169 ${ }^{+}$in the subcapsular sinus of the lymph nodes) have been shown to be crucially involved in crosspresentation of tumor antigens from apoptotic cells to $\mathrm{CD}^{+} \mathrm{T}$ cells $[16]$ and are also capable of presenting native antigens to $B$ cells $[17,18]$. However, the specific functions and further subtypes of antigen-presenting macrophages especially in humans remain largely unexplored. In the last years, it became evident that a variety of TRM, comprising alveolar macrophages in the lung, microglia in the brain, or Kupffer cells in the liver, are not only specifically adapted to maintain homeostasis and organ function, but are also derived from embryonic precursors early in development. Similar to studying the complex functions of specific DC subtypes, also the biology and function of these specific TRM are quite difficult to model with mature monocyte-derived macrophages.

\section{Generation and Immunomodulatory Properties of iPSC-Derived APCs}

One promising source for the generation of APCs for disease modeling or cell-based therapies are iPSCs (Fig. 1). iPSCs have been introduced in 2006 by Takahashi and Yamanaka [19], who could prove that the simple overexpression of the 4 transcription factors OCT4, SOX2, KLF4, and c-MYC can convert a somatic cell back into a pluripotent status. Over the past years, iPSCs have been generated from a variety of different cell types using several refined reprogramming techniques and have - given their unlimited proliferation and differentiation potential - been applied for disease modeling studies and drug screening approaches. Moreover, in the past years, iPSC derivatives found their way into first clinical studies for cell-based therapies [20,21]. So far, the generation of long-term engraftable hematopoietic stem cells (HSCs) remains hampered and has only been shown in a seminal study using a combinatory approach of a morphogendriven differentiation together with the overexpression of 7 different transcription factors (e.g., several HOX genes)
[22]. Whereas also the production of mature lymphocytes, such as B and T cells, remains challenging, the efficient differentiation of iPSCs into mature DCs or macrophages has been demonstrated by several groups [23].

The first generation of APCs derived from human PSCs, specifically embryonic stem cells (ESCs), was reported in 2004 by Zhan et al. [24]. The authors used a differentiation protocol based on the formation of embryoid bodies to induce undirected 3 germ layer differentiation and further supported hematopoietic specification and development by the addition of the hematopoietic cytokines SCF, FLT3 ligand, thrombopoietin, IL-3, GM-CSF, and IL-4. This rather unspecific protocol generated a variety of different $\mathrm{CD} 45^{+}$leukocytes, predominantly myeloid cells, including about $25 \%$ of HLA class $\mathrm{II}^{+}$and $\mathrm{CD}^{+} 6^{+}$macrophages and DCs. These HLA class $\mathrm{II}^{+}$APCs were able to stimulate purified allogeneic CD4 and CD8 $\mathrm{T}$ cells in mixed lymphocyte reactions.

Further studies generating more specific populations of DCs or macrophages were published following this report. Most of these protocols are either based on the formation of embryoid bodies or use a supportive/instructive co-culture with specific feeder cells (e.g., murine OP9 stromal cell) with or without the addition of mesoderm priming cytokines, such as BMP4, VEGF, and SCF. Subsequently, hematopoiesis is generally supported by the addition of hematopoietic cytokines, such as GM-CSF, M-CSF, and/or IL-4, depending on the desired output (see Table 1 for a summary of differentiation strategies).

\section{iPSC-Derived DCs}

For the generation of DCs from human ESCs, first refined protocols were introduced in 2006 and 2007 [25, 26]. Those 2 groups, however, focused on the development of protocols employing murine feeder cell-based co-culture strategies to induce hematopoietic development as already successfully applied before for the generation of DCs from murine PSCs [27]. Slukvin et al. [25] initiated differentiation by co-culture of human ESCs with murine OP9 stromal cells, followed by an expansion of myeloid cells with GM-CSF and - after purification using a density gradient centrifugation step - subsequent generation of DCs with GM-CSF and IL-4. Another study used a similar 3-step protocol specifying differentiation into DCs by the addition of GM-CSF, M-CSF, and IL-4, however, without the necessity for further purification steps [26]. The functionality of ESC-derived DCs was determined by cytokine secretion as well as the capacity to stimulate the proliferation of allogeneic $\mathrm{T}$ cells. Besides the use of murine feeder cells to support the hematopoietic differentiation of ESCs, improved embryoid bodybased protocols for the specific generation and maturation of iPSC-derived DCs have been introduced $[28,29]$. DCs generated by those 2 protocols showed a typical sur- 


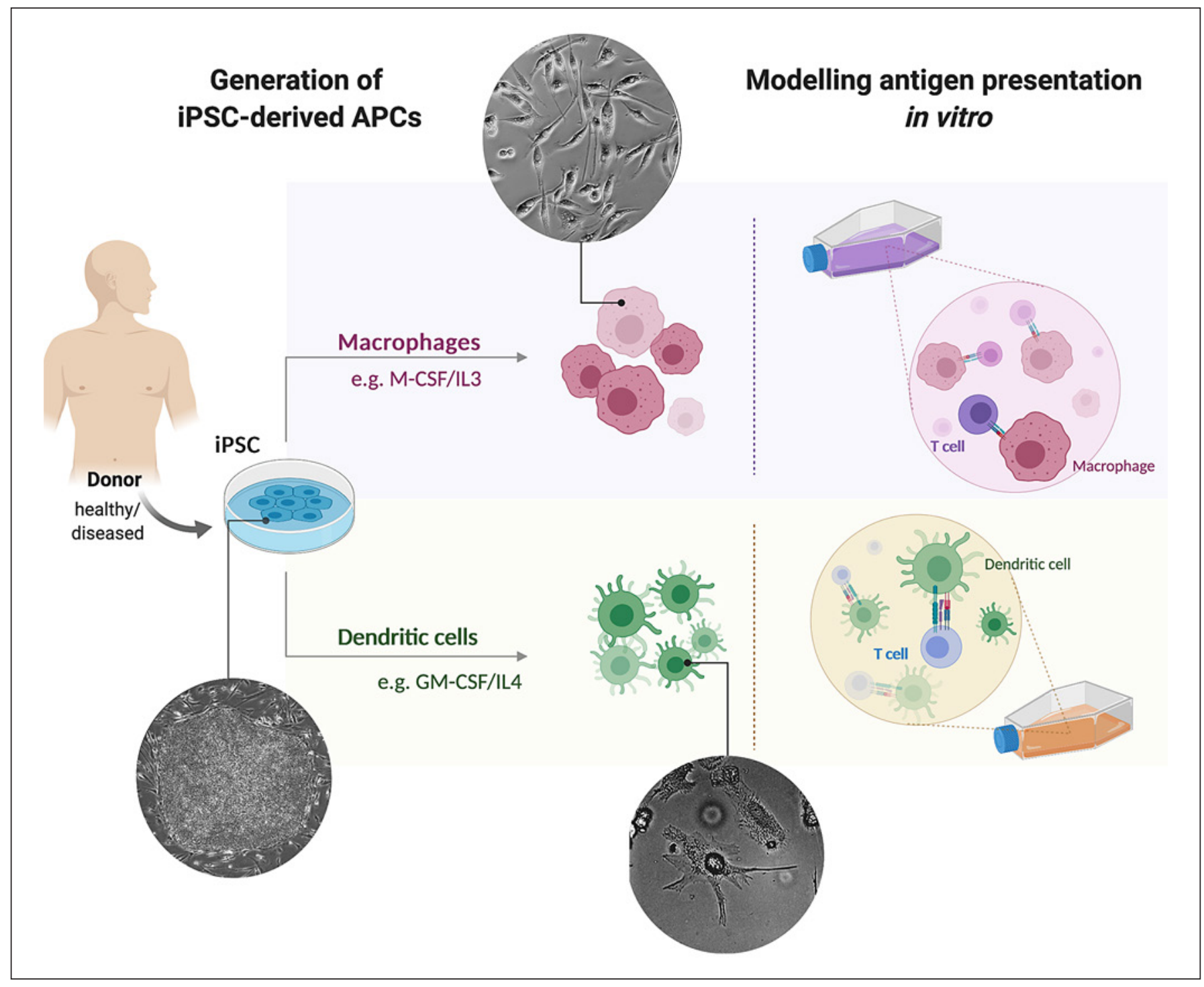

Fig. 1. Potential of induced pluripotent stem cells (iPSC)-derived antigen-presenting cells (APCs) for in vitro modeling of T-cell interactions. Left: Generation of iPSC-derived APCs: iPSC can be generated from easily assessable donor material, such as skin biopsies or blood samples, by overexpression of specific transcription factors. iPSC can be differentiated in vitro into different APCs, such as dendritic cells or macrophages, using specific lineage in- structive cytokines (e.g., IL-4 and GM-CSF or IL-3 and M-CSF, respectively). Right: Modeling antigen presentation in vitro: Subsequently, co-cultivation of the iPSC-derived APCs with autologous or allogeneic $\mathrm{T}$ cells derived from peripheral blood or iPSCs can be performed to study antigen presentation in vitro (created with BioRender.com). face phenotype and fulfilled critical biological functions, such as phagocytosis, chemotaxis, and cytokine/chemokine secretion (e.g., IL-12p70). Most importantly, ESCderived DCs also modulated allogeneic T-cell responses and were able to induce antiviral (Epstein-Barr virus and cytomegalovirus) and tumor antigen (melanoma antigen recognized by T cells 1 [MART-1] and telomerase reverse transcriptase [hTERT])-specific T-cell activation and expansion in a semi-allogenic context. Of note, both studies reported that the ESC-derived DCs showed a highly similar phenotype and function compared to peripheral blood moDCs.
Soon after the generation of human iPSCs, the established protocols were also utilized and further developed for the generation of iPSC-derived DCs [30, 31]. Importantly, studies applying iPSC-derived DCs now also offered the possibility to investigate their interaction with autologous $\mathrm{T}$ cells derived from the same donor/patient. Indeed, iPSC-derived DCs can stimulate not only allogeneic naïve $\mathrm{T}$ cells but also demonstrate efficient stimulation of autologous, antigen-specific $\mathrm{CD}^{+} \mathrm{T}$ cells after loading with a cytomegalovirus pp65 peptide [31]. Moreover, $\mathrm{CD} 8^{+} \mathrm{T}$ cells demonstrated efficient anti-tumor cytotoxicity when previously stimulated with autologous, 


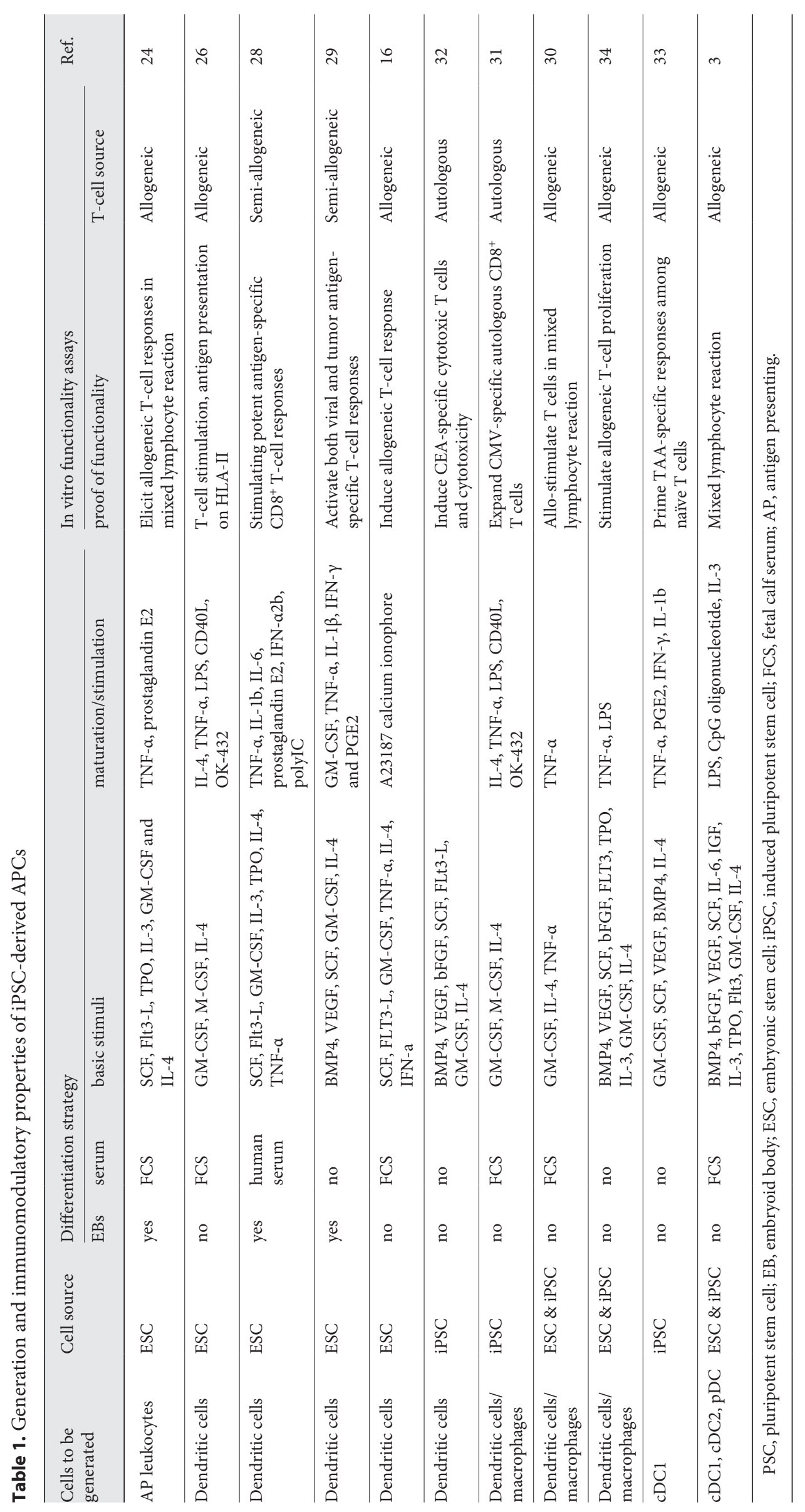


genetically modified, carcinoembryonic antigen-expressing iPSC-derived DCs [32]. Thus, these cells represent a highly attractive model system to study antigen presentation and T-cell priming by DCs in physiological and pathological settings. In the following years, differentiation protocols were further optimized to serum and feederindependent strategies, preparing for the requirements necessary for clinical translation of iPSC-DC-based cell therapies [29, 32-34].

Importantly, the generation of DCs from human PSCs in vitro also provides the possibility to finely modulate the differentiation process and generate rare and specific subsets of APCs with a defined immune-regulatory status. Indeed, iPSCs can give rise to defined $\mathrm{cDC} 1, \mathrm{cDC}$, and $\mathrm{pDC}$ subsets applying specific differentiation protocols. The generation of $\mathrm{CDC} 1$ cells characterized by the expression of chemokine receptor XCR1 and CD141 as well as the potential for cross-presentation and the induction of cytotoxic T cells was already demonstrated in 2012 [33] and further developed over the last years [35]. Moreover, a recent study demonstrates the generation of specific cDC1 (HLA-DR ${ }^{+}, \mathrm{CD} 11 \mathrm{c}^{+}, \mathrm{CD}_{14} 1^{+}, \mathrm{Clec} \mathrm{a}^{+}$), cDC2 $\left(\mathrm{HLA}_{-} \mathrm{DR}^{+}, \mathrm{CD} 11 \mathrm{c}^{+}, \mathrm{CD} 14^{-}, \mathrm{CD} 1 \mathrm{c}^{+}\right)$, and $\mathrm{pDC}(\mathrm{HLA}-$ $\mathrm{DR}^{+}, \mathrm{CD} 11 \mathrm{c}^{\text {low }}, \mathrm{CD} 123^{+}, \mathrm{CD} 33^{+}, \mathrm{CD} 34^{+}$) subsets from human iPSCs and applied this system to model and study the pathophysiology of IRF8 deficiency, a severe monocytic and DC immunodeficiency [3]. Especially these later studies further highlight the potential of iPSC technology for the generation of larger amounts of specific DC subsets to model (rare) diseases and provide and evaluate novel concepts for cell-based therapies.

\section{iPSC-Derived Macrophages}

Another interesting member of the class of professional APCs are macrophages. Similar to the generation of DCs, also the first protocols for the generation of monocytes/macrophages from human iPSCs can be grouped into protocols that are based on the formation of embryoid bodies or induce hematopoiesis by co-culture with stromal cells (also reviewed in $[11,21,36]$ ). However, the majority of currently applied protocols are based on the formation of embryoid bodies and induction of hematopoiesis by IL-3, M-CSF, or GM-CSF as described in 2008 by the team around William James [37]. Of note, here especially IL-3 seems to be an important cytokine and potent driver in iPSC-derived hematopoiesis [38]. In the last decade, this protocol has been further modified, adapted, and used for different in vitro studies [39-44]. As a prerequisite for the preclinical evaluation of iPSCderived macrophages in suitable mouse models and also a future translation of cell-based therapies, we further enhanced this protocol towards the continuous and scalable generation of iPSC-derived macrophages in industrycompatible stirred tank bioreactors [14]. The so gener- ated macrophages are similar to their peripheral bloodderived counterparts in terms of morphology, surface marker profile, phagocytosis, and bacterial killing. However, when looking at whole transcriptome analysis, iPSC-derived macrophages show a more primitive transcriptional fingerprint comparable to yolk sac-derived macrophages. Functionally, iPSC-derived macrophages have been used for disease modeling studies, in vitro infection experiments, as well as adoptive cell therapies, for example, for a rare pulmonary disease (pulmonary alveolar proteinosis [13]) or as a novel immunotherapy concept to target pulmonary infections [14]. Although the functionality of iPSC-derived macrophages in the context of cytokine secretion, chemotaxis, phagocytosis, as well as the antimicrobial activity has been studied in detail, the antigen-presenting properties of iPSC-derived macrophages remain mainly elusive. However, promising data has recently been provided by Joshi et al. [45] who showed that human iPSC-derived macrophages can indeed present antigens to autologous, islet-infiltrating $\mathrm{CD} 4^{+} \mathrm{T}$ cells of a diabetes type I patient. Moreover, our own unpublished results indicate that iPSC-derived macrophages are also able to present viral antigens and thereby expand autologous antigen-specific $\mathrm{CD} 8^{+} \mathrm{T}$ cells. The results further highlighting the applicability of iPSC-derived macrophages for disease modeling and cellular therapies based on APCs.

\section{Preclinical Assessment and Adoptive Transfer of (iPSC-Derived) APCs}

\section{Primary APCs}

DCs as well as macrophages are critical regulators of the immune system, as they are involved in both tissue homeostasis and the induction of tolerance, but also the initiation and coordination of protective immune responses in case of infection and inflammation. Given their pivotal roles in modulation of $\mathrm{T}$ - and $\mathrm{B}$-cell responses and, thus, the finetuning of the adaptive immune system, it does not come as a surprise that especially DCs but also macrophages have been used in several (pre-)clinical studies targeting a variety of diseases characterized by imbalanced or unappropriated immune responses. One very well-explored application of DC-based cellular therapies is immune-oncology and, specifically, the use of DC-based cellular vaccines to poorly immunogenic cancer antigens [46]. Similarly, also activated macrophages have been applied for the treatment of cancer patients in clinical studies [47]. On the other side, also therapeutic approaches applying APCs to modulate the immune system and create tolerance, for example, in the context of whole-organ transplantations or also type 1 diabetes, have been performed [48]. Although all these studies 
demonstrate a good safety profile of the adoptive cell transfer, the results in relation to clinical efficacy were quite heterogeneous [35].

One of the reasons underlying these partially disappointing outcomes might be attributed to the phenotype of DCs and macrophages employed in the clinical trials. Those cells were mainly derived from autologous patients' peripheral blood monocytes, which were further differentiated in vitro. This strategy, however, includes several problems. First, it is well appreciated that there is great heterogeneity between monocyte-derived macrophages and DCs derived from different donors. Moreover, a number of donors have previously been treated, for example, with anti-cancer chemotherapy, further impacting the quality and activation status of the isolated monocytes and, thus, also the generated cell product [49]. Finally, the specific cell identity might have a strong impact on its function and the clinical outcome. Especially in respect to an application in immune-oncology, moDCs have been described to have an inadequate capacity for cross-presentation of antigens to naïve $\mathrm{CD}^{+} \mathrm{T}$ cells and induce only insufficient cytotoxic $\mathrm{T}$-cell responses to the cancer cells [35].

\section{The Potential of iPSC-Derived APCs in Clinical \\ Translation}

One possibility to overcome those limitations and hopefully increase the success of cell-based therapies employing APCs could be the use of iPSC technology. As mentioned before, several groups have described the generation of iPSC-derived DCs and macrophages, which display classical morphology, surface marker profiles, and also functionality. Moreover, the in vitro generation from human iPSCs also allows for the derivation of rare, yet desirable, specialized populations of APCs. Importantly, Silk et al. [33] demonstrate that only their XCR ${ }^{+}$/ CD $141^{+}$iPSC-derived DCs could be used for cross-presentation of tumor-associated antigen to naïve $\mathrm{T}$ cells, but not classical XCR $1^{-}$moDCs.

Although iPSC-derived APCs have been mainly evaluated in complex co-culture systems in vitro due to complications with suitable xenograft models, murine iPSCderived APCs have shown therapeutic success in mouse models. For example, murine iPSC-derived regulatory DCs have been efficiently applied as an immunosuppressive vaccine to induce donor-specific regulatory $\mathrm{T}$ cells in a murine transplantation model for allogeneic cardiac grafts. This study even demonstrated a superior effect of iPSC-derived DCs when comparing them to bone marrow-derived DCs [50]. Similarly, Kitadani et al. [32] - after proving the functionality of their human iPSC-derived DCs in vitro - showed proof of concept in vivo studies for anti-cancer vaccination therapy using mouse iPSC-derived DCs in suitable mouse models.

\section{Hurdles for iPSC-Derived APCs in Clinical}

Translation

Besides all these advantages, the use of iPSC-derived cells in clinical practice also includes challenges. One critical point is the efficient upscaling of cultivation and differentiation processes, but also the purity of the generated cell product [21]. One more challenge could be a potentially immature phenotype of the iPSC derivatives. Indeed, it has been reported in several publications that one critical challenge for iPSC-based therapies is that the cells more closely resemble embryonic or neonatal cell types with subtle but potentially important differences. In the case of immune cells, those differences are mainly associated with the activation status. Some reports, for example, demonstrate that iPSC-derived DCs secrete more of the anti-inflammatory cytokine IL-10, a feature also observed in neonatal DCs and associated with tolerance induction [35]. Similarly, iPSC-derived macrophages show transcriptional similarities with embryonic yolk sac-derived macrophages - precursors of several tissue-resident macrophage populations [11]. Those cells have been described as more immunomodulatory, and our own studies show that also iPSC-derived macrophages secrete more IL-10 then their monocyte-derived counterparts [42]. These features may favor the application of iPSCderived APCs in diseases associated with hyperinflammation or transplantation settings with the aim of tolerance induction. However, we also recently examined the potential to use iPSC-derived macrophages as an immunotherapy approach to target pulmonary infections. The iPSC-derived cells were not only able to efficiently ameliorate disease-associated symptoms and bacterial load in a murine model for pulmonary Pseudomonas aeruginosa infections, but in vitro data also demonstrated that the iPSC-derived macrophages reacted with stronger pro-inflammatory transcriptional changes to pathogen contact and secreted higher amounts of pro-inflammatory cytokines when compared to monocyte-derived macrophages [14 and own unpubl. data]. These data indicate that despite a more primitive phenotype, iPSC-derived cells can still possess appropriate or even superior antimicrobial potential. Thus, it will be very exciting to study the effect of cell-based therapies employing iPSC-derived APCs in various disease entities.

\section{Conclusion}

APCs play a pivotal role in the modulation of $\mathrm{T}$ - and B-cell responses and the finetuning of the adaptive immune system. Thus, they hold great promise for cellbased immunotherapies. iPSC technology offers a reliable cell source to generate a plethora of DCs, macrophages, and other cells of the innate immune system. In this line, 
various studies could already demonstrate that iPSC-derived DCs and macrophages share critical hallmarks with their in vivo counterparts in respect to morphology and functionality. Given these similarities, iPSC from both healthy individuals and diseased patients have been used to gain insights into the immunomodulatory properties of iPSC derivatives and the underlying mechanism of the respective disease. In addition, preclinical studies using DCs and macrophages could already demonstrate that the adoptive transfer of these two cell types can have beneficial effects in defined immunotherapeutic applications. Hence, the technology of iPSC as an unlimited cell source and the possibility for their scalable cultivation and precise differentiation into rare yet desirable subsets may enhance the field of transfusion medicine. Although recent progress has been made in the scalable cultivation as well as differentiation of iPSC towards APCs, knowledge of critical attributes with respect to the developmental origin of iPSC-derived APCs or overall immunogenicity of the generated cells is still scarce and these issues need to be considered in future (pre-)clinical applications. Especially the latter is of great importance if thinking of an off-the-shelf cell product. While as of yet, the generation, establishment, and subsequent differentiation of iPSC towards APCs for an individual patient are very cost intensive, strategies to manipulate the HLA compartment to maintain, for example, the capacity for antigen presentation but to reduce allo-reactions at the same time may be the next logical step to enhance the clinical transition of iPSC-derived APCs.

\section{Conflict of Interest Statement}

The authors have no conflicts of interest to declare.

\section{Funding Sources}

This work was supported by grants from the Deutsche Forschungsgemeinschaft (Cluster of Excellence REBIRTH; Exc 62/3 to N.L., DFG LA 3680/2-1, DFG Research Unit 2830 [EI 493/1-1]), the German Ministry for Education and Science (iMACnet 01EK1602A), Else Kröner-Fresenius-Stiftung (EKFS; 2015_A92 to N.L. and EKFS; 2016_A146 to M.A.), and the Hannover Medical School Transplantation Center (Tx Center). This project has received funding from the European Research Council (ERC) under the European Union's Horizon 2020 research and innovation program (grant agreement No. 852178). The work also received funding from "Förderung aus Mitteln des Niedersächsischen Vorab."

\section{Author Contributions}

A.C.D.: manuscript writing, final approval of manuscript; M.A. and N.L.: conception/design, manuscript writing, final approval of manuscript.

\section{References}

1 Ugur M, Mueller SN. T cell and dendritic cell interactions in lymphoid organs: more than just being in the right place at the right time. Immunol Rev. 2019 May;289(1):115-28.

2 Merad M, Sathe P, Helft J, Miller J, Mortha A. The dendritic cell lineage: ontogeny and function of dendritic cells and their subsets in the steady state and the inflamed setting. Annu Rev Immunol. 2013;31(1):563-604.

3 Sontag S, Förster M, Qin J, Wanek P, Mitzka S, Schüler HM, et al. Modelling IRF8 Deficient Human Hematopoiesis and Dendritic Cell Development with Engineered iPS Cells. Stem Cells. 2017 Apr;35(4):898-908.

4 Collin M, Bigley V. Human dendritic cell subsets: an update. Immunology. 2018 May; 154(1):3-20.

5 Stripecke R. Lentivirus-Induced Dendritic Cells (iDC) for Immune-Regenerative Therapies in Cancer and Stem Cell Transplantation. Biomedicines. 2014 Aug;2(3):229-46.

6 Guo X, Zhou Y, Wu T, Zhu X, Lai W, Wu L. Generation of mouse and human dendritic cells in vitro. J Immunol Methods. 2016 May; 432:24-9.

7 Constantino J, Gomes C, Falcão A, Neves BM, Cruz MT. Dendritic cell-based immunotherapy: a basic review and recent advances. Immunol Res. 2017 Aug;65(4):798-810.
8 Scalavino V, Liso M, Serino G. Role of microRNAs in the Regulation of Dendritic Cell Generation and Function. Int J Mol Sci. 2020 Feb;21(4):E1319.

9 Hancock DG, Guy TV, Shklovskaya E, Fazekas de St Groth B. Experimental models to investigate the function of dendritic cell subsets: challenges and implications. Clin Exp Immunol. 2013 Feb;171(2):147-54.

10 van Helden SF, van Leeuwen FN, Figdor CG. Human and murine model cell lines for dendritic cell biology evaluated. Immunol Lett. 2008 May;117(2):191-7.

11 Lee CZ, Kozaki T, Ginhoux F. Studying tissue macrophages in vitro: are iPSC-derived cells the answer? Nat Rev Immunol. 2018 Nov; 18(11):716-25.

12 Ansprenger C, Amberger DC, Schmetzer HM. Potential of immunotherapies in the mediation of antileukemic responses for patients with acute myeloid leukemia (AML) and myelodysplastic syndrome (MDS) - With a focus on Dendritic cells of leukemic origin (DCleu). Clin Immunol. 2020 Aug;217:108467.

13 Happle C, Lachmann N, Škuljec J, Wetzke M, Ackermann M, Brennig S, et al. Pulmonary transplantation of macrophage progenitors as effective and long-lasting therapy for hereditary pulmonary alveolar proteinosis. Sci Transl Med. 2014 Aug;6(250):250ra113.
14 Ackermann M, Kempf H, Hetzel M, Hesse C, Hashtchin AR, Brinkert K, et al. Bioreactorbased mass production of human iPSC-derived macrophages enables immunotherapies against bacterial airway infections. Nat Commun. 2018 Nov;9(1):5088.

15 Pozzi LA, Maciaszek JW, Rock KL. Both dendritic cells and macrophages can stimulate naive CD8 T cells in vivo to proliferate, develop effector function, and differentiate into memory cells. J Immunol. 2005 Aug;175(4): 2071-81.

16 Asano K, Nabeyama A, Miyake Y, Qiu CH, Kurita A, Tomura M, et al. CD169-positive macrophages dominate antitumor immunity by crosspresenting dead cell-associated antigens. Immunity. 2011 Jan;34(1):85-95.

17 Carrasco YR, Batista FD. B cells acquire particulate antigen in a macrophage-rich area at the boundary between the follicle and the subcapsular sinus of the lymph node. Immunity. 2007 Jul;27(1):160-71.

18 Junt T, Moseman EA, Iannacone M, Massberg S, Lang PA, Boes M, et al. Subcapsular sinus macrophages in lymph nodes clear lymph-borne viruses and present them to antiviral B cells. Nature. 2007 Nov;450(7166): 110-4. 
19 Takahashi K, Yamanaka S. Induction of pluripotent stem cells from mouse embryonic and adult fibroblast cultures by defined factors. Cell. 2006 Aug;126(4):663-76.

20 Mandai M, Watanabe A, Kurimoto Y, Hirami Y, Morinaga C, Daimon T, et al. Autologous Induced Stem-Cell-Derived Retinal Cells for Macular Degeneration. N Engl J Med. 2017 Mar;376(11):1038-46.

21 Haake K, Ackermann M, Lachmann N. Concise Review: Towards the Clinical Translation of Induced Pluripotent Stem Cell-Derived Blood Cells-Ready for Take-Off. Stem Cells Transl Med. 2019 Apr;8(4):332-9.

22 Sugimura R, Jha DK, Han A, Soria-Valles C da Rocha EL, Lu YF, et al. Haematopoietic stem and progenitor cells from human pluripotent stem cells. Nature. 2017 May; 545(7655):432-8

23 Ackermann M, Liebhaber S, Klusmann JH, Lachmann N. Lost in translation: pluripotent stem cell-derived hematopoiesis. EMBO Mol Med. 2015 Nov;7(11):1388-402.

24 Zhan X, Dravid G, Ye Z, Hammond H, Shamblott M, Gearhart J, et al. Functional antigenpresenting leucocytes derived from human embryonic stem cells in vitro. Lancet. 2004 Jul;364(9429):163-71.

25 Slukvin II, Vodyanik MA, Thomson JA, Gumenyuk ME, Choi KD. Directed differentiation of human embryonic stem cells into functional dendritic cells through the myeloid pathway. J Immunol. 2006 Mar;176(5):292432.

26 Senju S, Suemori H, Zembutsu H, Uemura Y, Hirata S, Fukuma D, et al. Genetically manipulated human embryonic stem cell-derived dendritic cells with immune regulatory function. Stem Cells. 2007 Nov;25(11):2720-9.

27 Senju S, Hirata S, Matsuyoshi H, Masuda M, Uemura Y, Araki K, et al. Generation and genetic modification of dendritic cells derived from mouse embryonic stem cells. Blood. 2003 May;101(9):3501-8.

$28 \mathrm{Su}$ Z, Frye C, Bae KM, Kelley V, Vieweg J. Differentiation of human embryonic stem cells into immunostimulatory dendritic cells under feeder-free culture conditions. Clin Cancer Res. 2008 Oct;14(19):6207-17.

29 Tseng SY, Nishimoto KP, Silk KM, Majumdar AS, Dawes GN, Waldmann H, et al. Generation of immunogenic dendritic cells from human embryonic stem cells without serum and feeder cells. Regen Med. 2009 Jul;4(4):51326.
30 Choi KD, Vodyanik MA, Slukvin II. Generation of mature human myelomonocytic cells through expansion and differentiation of pluripotent stem cell-derived linCD34+CD43+CD45+ progenitors. J Clin Invest. 2009 Sep;119(9):2818-29.

31 Senju S, Haruta M, Matsumura K, Matsunaga Y, Fukushima S, Ikeda T, et al. Generation of dendritic cells and macrophages from human induced pluripotent stem cells aiming at cell therapy. Gene Ther. 2011 Sep;18(9):874-83.

32 Kitadani J, Ojima T, Iwamoto H, Tabata H, Nakamori M, Nakamura M, et al. Cancer Vaccine Therapy Using Carcinoembryonic Antigen - expressing Dendritic Cells generated from Induced Pluripotent Stem Cells. Sci Rep. 2018 Mar;8(1):4569.

33 Silk KM, Silk JD, Ichiryu N, Davies TJ, Nolan KF, Leishman AJ, et al. Cross-presentation of tumour antigens by human induced pluripotent stem cell-derived CD141(+)XCR1+ dendritic cells. Gene Ther. 2012 Oct;19(10): 1035-40.

34 Yanagimachi MD, Niwa A, Tanaka T, Honda-Ozaki F, Nishimoto S, Murata Y, et al. Robust and highly-efficient differentiation of functional monocytic cells from human pluripotent stem cells under serum- and feeder cell-free conditions. PLoS One. 2013; 8(4):e59243.

35 Sachamitr P, Leishman AJ, Davies TJ, Fairchild PJ. Directed Differentiation of Human Induced Pluripotent Stem Cells into Dendritic Cells Displaying Tolerogenic Properties and Resembling the CD141+ Subset. Front Immunol. 2018 Jan; 8:1935.

36 Rajab N, Rutar M, Laslett AL, Wells CA. Designer macrophages: pitfalls and opportunities for modelling macrophage phenotypes from pluripotent stem cells. Differentiation. 2018 Nov-Dec;104:42-9.

37 Karlsson KR, Cowley S, Martinez FO, Shaw M, Minger SL, James W. Homogeneous monocytes and macrophages from human embryonic stem cells following coculture-free differentiation in M-CSF and IL-3. Exp Hematol. 2008 Sep;36(9):1167-75.

38 Ackermann M, Haake K, Kempf H, Kaschutnig P, Weiss AC, Nguyen AH, et al. A 3D iPSC-differentiation model identifies interleukin-3 as a regulator of early human hematopoietic specification. Haematologica. 2020 Apr;haematol.2019.228064.

39 van Wilgenburg B, Browne C, Vowles J, Cowley SA. Efficient, long term production of monocyte-derived macrophages from human pluripotent stem cells under partly-defined and fully-defined conditions. PLoS One. 2013 Aug;8(8):e71098.
40 Lachmann N, Happle C, Ackermann M, Lüttge D, Wetzke M, Merkert S, et al. Gene correction of human induced pluripotent stem cells repairs the cellular phenotype in pulmonary alveolar proteinosis. Am J Respir Crit Care Med. 2014 Jan;189(2):167-82.

41 Hale C, Yeung A, Goulding D, Pickard D, Alasoo K, Powrie F, et al. Induced pluripotent stem cell derived macrophages as a cellular system to study salmonella and other pathogens. PLoS One. 2015 May;10(5):e0124307.

42 Lachmann N, Ackermann M, Frenzel E, Liebhaber S, Brennig S, Happle C, et al. Largescale hematopoietic differentiation of human induced pluripotent stem cells provides granulocytes or macrophages for cell replacement therapies. Stem Cell Reports. 2015 Feb;4(2): 282-96.

43 Buchrieser J, James W, Moore MD. Human Induced Pluripotent Stem Cell-Derived Macrophages Share Ontogeny with MYB-Independent Tissue-Resident Macrophages. Stem Cell Reports. 2017 Feb;8(2):334-45.

44 Yeung AT, Hale C, Lee AH, Gill EE, Bushell W, Parry-Smith D, et al. Exploiting induced pluripotent stem cell-derived macrophages to unravel host factors influencing Chlamydia trachomatis pathogenesis. Nat Commun. 2017 Apr;8(1):15013.

45 Joshi K, Elso C, Motazedian A, Labonne T, Schiesser JV, Cameron F, et al. Induced pluripotent stem cell macrophages present antigen to proinsulin-specific $\mathrm{T}$ cell receptors from donor-matched islet-infiltrating $\mathrm{T}$ cells in type 1 diabetes. Diabetologia. 2019 Dec; 62(12):2245-51.

46 Wculek SK, Cueto FJ, Mujal AM, Melero I, Krummel MF, Sancho D. Dendritic cells in cancer immunology and immunotherapy. Nat Rev Immunol. 2020 Jan;20(1):7-24.

47 Klimp AH, de Vries EG, Scherphof GL, Daemen T. A potential role of macrophage activation in the treatment of cancer. Crit Rev Oncol Hematol. 2002 Nov;44(2):143-61.

48 Thomson AW, Ezzelarab MB. Regulatory dendritic cells: profiling, targeting, and therapeutic application. Curr Opin Organ Transplant. 2018 Oct;23(5):538-45.

49 Leishman AJ, Silk KM, Fairchild PJ. Pharmacological manipulation of dendritic cells in the pursuit of transplantation tolerance. Curr Opin Organ Transplant. 2011 Aug;16(4): 372-8.

50 Cai S, Hou J, Fujino M, Zhang Q, Ichimaru N, Takahara S, et al. iPSC-Derived Regulatory Dendritic Cells Inhibit Allograft Rejection by Generating Alloantigen-Specific Regulatory T Cells. Stem Cell Reports. 2017 May; 8(5):1174-89. 